\title{
Risperidone improves severe tardive dyskinesia in people with schizophrenia
}

Bai Y-M, Yu S-C, Lin C-C. Risperidone for severe tardive dyskinesia: a 12-week randomized, double-blind, placebo-controlled study. J Clin Psychiatry 2003;64:1342-8.

\section{Q Does risperidone improve severe tardive dyskinesia in people with schizophrenia after conventional antipsychotics have been discontinued?}

\section{METHODS}

+

Design: Randomised controlled trial.

Allocation: Concealed.

Blinding: Double blind.

Follow up period: Twelve weeks.

Setting: Hospital based, Taiwan; timeframe not stated.

Patients: Forty nine people (aged 18-65 years; $66.7 \%$ male), hospitalised with chronic schizophrenia (DSM-IV) and severe tardive dyskinesia (mean Abnormal Involuntary Movement Scale (AIMS) score 15.9), maintained with conventional antipsychotics for $>1$ year with no record of aggressive or violent behaviour for 6 months and scoring $<20$ on the Brief Psychiatric Rating Scale (BPRS). Exclusions: major physical illness, prescribed atypical antipsychotics, or neuroleptic depot administration within preceding 6 months.

Intervention: Four week washout of conventional antipsychotics followed by risperidone $(2 \mathrm{mg} /$ day for 1 week, increased by $2 \mathrm{mg} /$ week to $6 \mathrm{mg} /$ day and maintained at this dose for the remainder of the trial); or placebo.

Outcomes: Tardive dyskinesia (AIMS), extrapyramidal side effects (extrapyramidal symptom rating scale (ESRS)), and psychiatric symptoms (BPRS).

a Patient follow up: $86 \%$

\section{MAIN RESULTS}

Risperidone significantly reduced tardive dyskinesia compared with placebo over 12 weeks (mean decrease in AIMS score: -5.5 with risperidone $v-1.1$ with placebo, $\mathrm{p}=0.001$ ). The differences between groups became significant at the end of the 8 th week onwards. $68 \%$ of the risperidone group responded, compared with $30 \%$ of the placebo group $(p=0.029)$. There was no significant difference in BPRS or ESRS scores after 12 weeks (see table). Improvement in tardive dyskinesia was mainly in the orofacial areas.

\section{CONCLUSIONS}

Risperidone significantly improves severe tardive dyskinesia caused by long term antipsychotic use in people with schizophrenia.

For correspondence: Chao-Cheng Lin Department of Psychiatry $\ddot{Y}_{u-L}$ Veterans Hospital, 91 Hsin Hsing St. Yu-Li, Hua-Lien, Taiwan; ymbi@ ms1.hinet.net

Sources of funding: Jansenn-Cilag Taiwan and Johnson \& Johnson Taiwan Ltd.
Table Mean change in total AIMS and ESRS scores at 12 weeks

\begin{tabular}{llll}
\hline Score & Risperidone & Placebo & p Value \\
\hline AIMS & -5.5 & -1.1 & 0.001 \\
Parkinsonian-ESRS & -0.5 & -0.6 & NS \\
Dystonia-ESRS & +0.4 & +0.5 & NS \\
BPRS & +1.5 & +5.3 & NS \\
\hline NS, not significant. & & & \\
\hline
\end{tabular}

Commentary

7

ardive dyskinesia is a serious and potentially irreversible complication of antipsychotic treatment with an annual cumulative incidence of $4 \%-5 \%$ and a 10 year prevalence of $15 \%-20 \%$ in young adults treated with first generation "typical" antipsychotics. One year studies indicate that second generation "atypical" antipsychotics (clozapine, risperidone, amisulpride, olanzapine, quetiapine, ziprasidone, aripiprazole) are associated with an $80 \%-90 \%$ lower risk of tardive dyskinesia. In contrast to the relative wealth of data indicating a lower risk of tardive dyskinesia with atypical rather than typical antipsychotic treatment, the utility of atypical antipsychotics in treating tardive dyskinesia is less clear. Whereas several studies show that clozapine effectively treats tardive dyskinesia (about $50 \%$ of patients respond), data with other atypical antipsychotics are less robust. Ya-Mei Bai and colleagues' report of a significant improvement in tardive dyskinesia in the course of 12 weeks' risperidone treatment is therefore of great interest. The fact that this improvement was most evident from week 8-12 of risperidone treatment suggests that the change more likely reflects a true antidyskinetic effect rather than a mere suppression or masking of tardive dyskinesia; this is corroborated by the absence of any relation between parkinsonism (EPS) and AIMS (tardive dyskinesia) scores.

Although in need of replication and elaboration, findings of this study do suggest a possible role for risperidone in the treatment of tardive dyskinesia. How risperidone compares with other atypical antipsychotics in this regard is unclear; particularly the question of whether it is as good as clozapine with regard to its antidyskinetic effect is not known. Consequently, clozapine remains the antipsychotic "gold standard" for the treatment of tardive dyskinesia pending additional study.

The primary defining characteristic of atypical agents is their reduced tendency to cause parkinsonian side effects and it is this property that principally explains their benefits in terms of a broader spectrum of efficacy and a lower risk of tardive dyskinesia. ${ }^{12}$ As a corollary, all atypical antipsychotics should be used properly (that is, produce an optimal antipsychotic effect without EPS or use of an anticholinergic agent) in order to provide the broader spectrum of efficacy and a lower risk of tardive dyskinesia; suitable patient selection and appropriate dosing are the key in accomplishing "atypical antipsychotic" behaviour and resultant benefits.

Rajiv Tandon, MD Chief Psychiatrist, State of Florida, Professor of Psychiatry, Institute of Health and Human Research, School of Social Work, Florida State University

1 Tandon R, Jibson MD. Efficacy of newer generation antipsychotics in the treatment of schizophrenia. Psychoneuroendocrinology 2003;28(Suppl 1): 9-26.

2 Tandon R, Jibson MD. Extrapyramidal side effects of antipsychotic treatment: scope of problem and impact on outcome. Ann Clin Psychiatry 2002;14:123-9. 